\title{
Un formulario de problemas mejora la comunicación entre pacientes y médicos
}

\author{
Patient completed schedule forms improves comunication between patients and doctors
}

Middleton JF et al.BMJ. 2006;332:1238-42

\section{Objetivo}

Evaluar el efecto sobre el resultado de la consulta médica, de traer un formulario que identifique los problemas de interés para el paciente (agenda) y de un taller educativo para médicos.

\section{Diseño}

Estudio multicéntrico, aleatorizado y controlado.

\section{Lugar}

Centros de Atención Primaria en el Reino Unido.

\section{Participantes}

46 médicos generalistas y 976 pacientes que pidieron turno con alguno de los médicos del estudio.

\section{Intervención}

Los médicos fueron aleatorizados a participar o no de un taller educativo de un día de duración que les permitiera adquirir habilidades en la identificación de ideas, preocupaciones, expectativas y forma de razonamiento de los pacientes, reconocer diferencias con su propia agenda y negociar un plan de acción común; practicaron con pacientes simulados, recibieron información teórica y retroalimentación sobre su desempeño. Se aleatorizó a los pacientes a recibir antes de la consulta un formulario que debían completar con los problemas de interés (agenda) y entregárselo al médico al entrar en la consulta (ensayo aletaorizado anidado de tipo agrupado*).

\section{Medición de resultados}

Número de problemas identificados y no identificados, tiempo requerido para manejarlos, duración de la consulta y satisfacción de los pacientes.

\section{Resultados}

Se contó con datos del $98 \%$ de los médicos aleatorizados y de $88 \%$ de los pacientes. El promedio de problemas identificados en cada consulta aumentó en 0,2 (IC95\%; 0,1 a 0,4) problemas identificados con la agenda del paciente; en $0,3(0,1$ a 0,6$)$ con el taller educativo, y en 0,5 (IC95\%: 0,3 a 0,7 ) con ambas intervenciones combinadas. No se encontraron diferencias en el tiempo requerido para manejar cada problema. La duración de la consulta aumentó 0,9 minutos (IC95: 0,3 a 1,5) con la agenda y 1,9 minutos (IC95\%; 1,0 a 2,8) con ambas intervenciones. La satisfacción del paciente sobre la relación médico-paciente aumentó significativamente sólo con la agenda preestablecida.

\section{Conclusión}

El uso de un formulario que identifique los problemas de interés para el paciente y de un taller educativo para médicos permite identificar más problemas en la consulta, prolongándolas mínimamente.

Palabras clave: Relación Médico-Paciente-Comunicación-Agenda.

Key words: doctor-patient relationship, communication, schedule.

Fuente de financiamiento: Beca del Colegio Real de Médicos Generales de Gran Bretaña.
Comentario

La consulta médica es la base de la práctica clínica y cualquier intervención que pueda mejorar su calidad tiene el potencial de mejorar todos los aspectos del cuidado de la salud. Si bien la capacidad para identificar el real motivo de consulta del paciente es un objetivo clave, muchas veces permanece oculto para el profesional de la salud. Los pacientes, a su vez, pueden sentir que no han podido discutir sus preocupaciones o que han recibido prescripciones o derivaciones no deseadas. En estos casos podemos decir que el proceso de la comunicación no ha resultado efectivo. Las intervenciones pueden realizarse desde el entrenamiento comunicacional de los médicos o ayudando a los pacientes a expresarse durante la consulta. En estudios previos, intervenciones educativas han demostrado tener efectos beneficiosos sobre el desempeño de los médicos ${ }^{12}$, pero pocos han demostrado una mejoría en el resultado de la consulta con pacientes reales ${ }^{3}$.

Los resultados de este trabajo indican que si se estimula a los pacientes a presentar una agenda más explícita, los médicos identifican más problemas, si bien las consultas duran algo más. Además, los pacientes que completaron una agenda estaban más satisfechos que los controles. Pero debemos tener en cuenta que la magnitud de la diferencia de problemas identificados es mínima y es discutible su relevancia clínica. Otro punto a considerar es que los médicos participantes eran voluntarios y no se puede asumir que estos hallazgos puedan extrapolarse a todos los médicos en general. Por otra parte, el efecto no parece tan claro con la intervención educativa a los médicos. Se desconoce si un entrenamiento educativo más intensivo podría llegar a lograr efectos de mayor magnitud a los hallados en este estudio y si la identificación de más problemas por consulta resultaría en mejores resultados de salud y mejor utilización de recursos. El mérito de este trabajo reside en el foco de estudio, la relación médico-paciente, punto extremadamente difícil de evaluar. Los autores han hecho un esfuerzo por cuantificar resultados y eso es encomiable, aunque quizás se necesiten otros métodos de estudio como el cualitativo, para investigar este tema. De hecho, un estudio reciente ${ }^{4}$ que utilizó el mismo diseño que el comentado aquí, no pudo demostrar diferencias en relación a la prescripción de fármacos, adherencia a los mismos y satisfacción de los pacientes que utilizaron una agenda escrita antes de la consulta.

\section{Conclusión de las comentadoras}

Este trabajo puede servir de base para realizar nuevos estudios centrados en la satisfacción de los pacientes, utilizando una intervención simple y poco costosa como la agenda preestablecida de los pacientes antes de ingresar a la consulta.

Sofa Catoggio y Victoria Wurcel [ Servicio de Medicina Familiar y Preventiva. Hospital Italiano de Buenos Aires. ]

Catoggio S., Wurcel V. Un formulario de problemas mejora la comunicación entre pacientes y médicos. Evid.Actual.Práct.Ambul.10 (2).43 MarzoAbril.2007.Comentado de Middleton JF et al.Effect of patient completed agenda forms and doctors' educationabout the agenda on the outcome of consultations: randomised controlled trial.BMJ. 2006 May 27;332(7552):1238-42. PMID: 16707508

\section{Referencias}

1.Stewart MA. Effective physician-patient communication and health outcomes - a review. Can Med Assoc J 1995;152:1423-33.

2. Hulsman RL, Ros WJ, Winnubst JA, Bensing JM. Teaching clinically experienced physicians communication skills. A review of evaluation studies. Med Educ 1999;33:655-68.

3. Evans BJ, Kiellerup FD, Stanley RO, Burrows GD, Sweet B. A communication skills programme for increasing patients' satisfaction with general practice consultations. Br J 3. Evans BJ, Kiellerup FD, Stanley RO, Burrows GD, Sweet B. A communication skills programme for increasing patients' satisfaction with general practice consultations. Br $J$
Med Psychol 1987;60:373-8.

4. Hamilton W. et al.The effect of patient self-completion agenda forms on prescribing and adherence in general practice: a randomized controlled trial.Fam Pract. 2006 Nov 30 .Epub. 